\title{
The Iced Drink Test - A Bedside Test for Diagnosis of Bulbar Myasthenia
}

\section{Nishit Sawal ${ }^{*}$}

Department of Medicine, Government Medical College and Hospital-Sector 32, Chandigarh, India

\begin{abstract}
The diagnosis of Myasthenia gravis (MG) is chiefly based on clinical symptoms and neurological examination coupled with serological testing for the presence of auto-antibodies, electrodiagnostic studies consisting of repetitive nerve stimulation test (RNST) and single fiber electromyography (SFEMG) and pharmacologic tests such as the edrophonium test. The ice pack test, based on the scientific principle that cooling improves neuromuscular transmission in Myasthenia gravis (MG), is a low cost, sensitive and specific test used for differentiating myasthenic ptosis from other causes of ptosis.

Based on the same principle, we propose the iced drink test as a quick, bedside test for differentiating bulbar weakness caused by myasthenia gravis from other causes of lower motor neuron type of bulbar weakness. The iced drink test, if validated, can prove to be useful in quick diagnosis of bulbar myasthenia.
\end{abstract}

Keywords: Bulbar myasthenia; Diagnose; Electrophysiological testing; Ice pack; Safe bedside test

\section{Introduction}

Myasthenia gravis (MG) is the most frequently encountered neuromuscular junction (NMJ) disorder in clinical practice with a prevalence of 5 to 15 cases per 100,000 [1]. It is an autoimmune disease caused by antibodies directed against the nicotinic acetylcholine receptors $(\mathrm{AChR})$ on the post synaptic membrane in $70 \%$ to $80 \%$ of patients [2] and in up to $40 \%$ of those patients negative for AChR antibodies, antibodies against the muscle specific Kinase (MuSK) can be demonstrated [3]. These antibodies produce impairment of normal NMJ function causing reduced or blocked transmission from the nerve to the muscle, producing weakness and fatigability.

Ocular symptoms in form of ptosis and/or diplopia are the presenting sign of the disease in $50 \%$ to $75 \%$ of patients [2]. Myasthenic patients can also present with isolated bulbar symptoms $(20.73 \%)$ or bulbar plus ocular symptoms (23.17\%). The voice can be affected with palatal weakness causing nasal voice and laryngeal weakness producing dysphonia. Dysarthria and dysphagia are seen, especially with tongue and/or lower facial weakness. Chewing weakness with difficulty chewing hard foods and fatigue with prolonged chewing is seen [4-6].

The diagnosis of MG is predominantly clinical aided by the presence of serum antibodies (e.g. AChR, MuSK) or abnormal electrodiagnosticstudies which consist of repetitive nerve stimulation test (RNST) and single fiber electromyography (SFEMG). Another commonly used test is the edrophonium test which consists of injecting a short-lived inhibitor of acetylcholinesterase (AChE) to increase the availability of acetylcholine at the NMJ. This produces a quick and often dramatic improvement in the symptoms or signs of MG (e.g. ptosis, dysphonia) [7]

Another commonly used bedside test is the ice pack test for ptosis. The test consists of the application of ice to the eyes for 2-5 min with myasthenic ptosis improving after cooling. The sensitivity and specificity of the ice pack test in ocular myasthenia associated or not with generalized symptoms has been reported in various studies as varying from $80 \%$ to $100 \%$ [8-11]. Using the same principle of improvement in neuromuscular transmission at the neuromuscular junction (NMJ) with cooling, we propose a novel test - the iced drink test for bedside diagnosis of bulbar myasthenia.

\section{Case Report}

A 42-year-old male, working as a college lecturer presented to Neurology OPD with symptoms of dysphonia and dysphagia which were of gradual subacute onset since the past 8-10 days. Patient had noticed he had difficulty eating digestive biscuits (biscuits which contain wheat flour/bran/fiber and added roughage and are claimed by manufacturers to have less calories than the regular ones), he felt as if they got stuck in his throat and he had to wash them down with a sip of water. His wife had noticed a slight nasal twang to his voice since the same duration and slight lowering of loudness of voice. Patient had no chewing difficulty (He had eaten chicken thrice during the mentioned period without any difficulty), no ptosis, diplopia or neck weakness and had not noticed a diurnal pattern to his symptoms and did not report fatigability. Patient was a non-diabetic, non-hypertensive, non smoker and there was no history of any other substance abuse. There was no history of recent insect bite or vaccination. There was no history of any throat illness. Past medical history was unremarkable. Family history was unremarkable.

On neurological examination, tone and power was normal in all muscles, tongue power tested by having patient push his cheek from inside against resistance) was normal, tongue tone was normal. All reflexes were normal, jaw jerk was absent, gag reflex was slightly diminished and there was a slight nasal twang to his voice (Since the neurologist was seeing the patient for the first time, this was more on testimony of his wife and sister-the nasal twang was not very marked). There was no tongue atrophy and no fasciculations. Rest of neurological examination was unremarkable.

A provisional clinical diagnosis of subacute onset lower motor neuron (LMN) type bulbar pathology was made and differential diagnosis's entertained as per neural axis localization were at level of

*Corresponding author: Nishit Sawal, Assistant Professor, Department of Medicine, Government Medical College and Hospital-Sector 32, Chandigarh, India, Tel: 0172-2601024; E-mail: drnishitsawal@gmail.com

Received March 06, 2017; Accepted March 16, 2017; Published March 21, 2017

Citation: Sawal N (2017) The Iced Drink Test - A Bedside Test for Diagnosis of Bulbar Myasthenia. J Neurol Disord 5: 333. doi:10.4172/2329-6895.1000333

Copyright: (c) 2017 Sawal N. This is an open-access article distributed under the terms of the Creative Commons Attribution License, which permits unrestricted use, distribution, and reproduction in any medium, provided the original author and source are credited. 
nerve (polyneuritis cranialis) and NMJ (Mysathenia gravis). Initially a formal $30 \mathrm{ml}$ water swallow test of a patient was carried out [12]. This was normal and he could easily gulp down water without any regurgitation or choking. Patient's voice was evaluated by making his wife stand at 15 meters from the patient in the hospital corridor and his younger sister at 30 meters and the patient was asked to say certain words (10 words) and some short sentences. His wife and sister were asked to evaluate the loudness and nasal twang of his voice. Patient was then given a glass of iced water, more than half of glass was filled with crushed ice and rest with cold water and patient was asked to sip it, swirl in his mouth and also do gargles with the iced water. He was asked to do so for 4-5 min and then he was again asked to say the same words (10 words) and same short sentences. His wife and sister again evaluated the loudness and nasal twang of his voice at 15 and 30 meters.

Post the iced water intervention, the patient, his wife and sister all concurred that there was a significant improvement in both the parameters being evaluated. There was an increase in loudness and the nasal twang disappeared. We also noticed that his nasal twang almost disappeared post the iced drink test only to reappear after some time. Keeping the possibility of myasthenia now on top of differential diagnosis's, an acetylcholine receptor antibody assay was obtained which came out to be positive. The patient refused to give consent for both the repetitive nerve stimulation test (RNST) as well as the edrophonium test. MRI-brain and cervical spine was done to exclude any mimics and was normal. Laryngoscopic examination was normal. Chest X-ray was normal. CT-Thorax is duly scheduled. Patient was put on pyridostigmine and corticosteroids and reported improvement of symptoms with treatment. Next follow up with CT-Thorax is due shortly. Further therapeutic plan is to assess patient for thymectomy and to taper off steroids and add appropriate steroid sparing agent.

\section{Discussion}

Clinical history and neurological examination form the bedrock of diagnosis of myasthenia gravis. In addition, tests used to confirm the clinical diagnosis include the edrophonium test, electrophysiological studies, ice pack test for myasthenic ptosis and serological tests for auto-antibodies [7]. The icepack test has a sensitivity and specificity of $80 \%$ to $100 \%$ [8-11]. It is a cheap, safe, quick to perform useful bedside test with no false-positives reported [11]. Studies showed improved neuromuscular junction transmission in myasthenia with cooling [1315]. This is owing to lesser acetylcholinesterase activity at temperatures below $28^{\circ} \mathrm{C}$, providing increasing amount of acetylcholine molecules in the synaptic cleft by decreasing the hydrolysis rate of acetylcholine molecules in the synaptic cleft [15]. Based on these studies, local cooling by application of ice pack was proposed as a simple procedure for the diagnosis of ocular myasthenia [16]. Numerous subsequent studies have confirmed the diagnostic validity of this test in the evaluation of ocular myasthenia which has been found to have a high sensitivity and specificity of around $80 \%$ to $100 \%$ [8-11].

The currently used diagnostic tests for myasthenia other than the serological tests for auto-antibodies are invasive and have certain disadvantages. The edrophonium test can cause complications like cardiac arrhythmias, respiratory failure and seizures. Resuscitation facilities should always be available while carrying out the test and requires continuous patient monitoring $[17,18]$. The electrodiagnostic studies which consist of repetitive nerve stimulation test (RNST) and single fiber electromyography (SFEMG) also have their limitations. SFEMG is the most sensitive technique but it is invasive and employs a needle electrode, requires special equipment and training and is timeand labor-intensive $[19,20]$, and is not widely available in developing countries. SFEMG also has the potential of causing hemorrhage, hematoma formation and infection. Since repetitive nerve stimulation test (RNST) is a painful procedure which involves supramaximal stimulation of a peripheral nerve to measure compound muscle action potential (CMAP) decrement with repetitive stimuli [21], it is poorly tolerated by some patients.

\section{Conclusion}

We have used the well known principle of cooling improving neuromuscular transmission in myasthenia to devise the iced drink test for bulbar myasthenia. We propose that the test be done only after carrying out a formal swallow test of the patient. If the patient fails the swallow test, the iced drink should not be carried out otherwise the patient may aspirate. If the patient has a normal swallow test, then only the iced drink test should be carried out. First a baseline evaluation of patient's symptoms should be done so as to allow a rough objectification. Since our patient had reported a nasal twang with some lowering of loudness of voice, these were the parameters we chose to assess. The patient should be given a glass of iced water filled more than half with crushed ice. The patient should then be asked to sip it, swirl it in his mouth and to do gargles with the iced water so as to subject the oral cavity, the hard and soft palate and the pharynx to contact with ice cold water so as to cause local cooling for 4-5 min. Post the iced drink, the patient can again be asked to do the same activity done prior to the administration of the iced drink and improvement, if any should be noted. In developing countries where neurologists are very few [22], and have very busy OPD's catering to a large number of patients every day, a simple, rapid, non-invasive, inexpensive test for rapid diagnosis of myasthenia can prove to be very useful. In addition, in developing countries electrodiagnostic studies like RNST and SFEMG are usually available only in select centers, are expensive tests and usually come with a long waiting period. Moreover invasive tests like the edrophomium test which involve a short term hospital admission and can cause serious side effects are also cumbersome to carry out. In this scenario, the iced drink test, if it proves to be of value in MG in a properly designed study, can prove to be of value. The iced drink test may be helpful to guide the next line of investigations. eg. If a patient presenting with bulbar symptoms has a positive iced water test, one can straightway proceed to a RNST/SFEMG and serological testing for AChR antibodies with higher degree of confidence. I propose this novel iced drink test as a quick, safe, inexpensive way to diagnose bulbar myasthenia. More studies however are needed before this test can be accepted in clinical practice.

\section{References}

1. Robertson NP, Deans J, Compston DA (1998) Myasthenia gravis: A population based epidemiological study in Cambridgeshire, England. J Neurol Neurosurg Psychiatry 65: 492-496.

2. Luchanok V, Kaminski HJ (2012) Natural history of myasthenia gravis. In Myasthenia gravis and myasthenia disorders. (2nd edn), Oxford University Press, Oxford, New York.

3. McConville J, Farrugia ME, Beeson D, Kishore U, Metcalfe R, et al. (2004) Detection and characterization of MuSK antibodies in seronegative myasthenia gravis. Annals of Neurology, 55: 580-584.

4. Kurt B, Ebru C, Asli K, Erdem OS, Rana K, et al. (2013) Myasthenia Gravis Single entity variable clinical features: Ten years of clinical experience in a tertiary care center ten years clinical experience of a tertiary care center. $\mathrm{J}$ Neurol Sci (Turk) 30: 135-143.

5. Sharp HR, Degrip A, Mitchell DB, Heller A (2001) Bulbar presentations of myasthenia gravis in the elderly patient. J Laryngol Otol 115: 1-3.

6. Chang CH, Lee KW, Kuo WR (2004) Dysphonia as the initial symptom of myasthenia gravis. J Otolaryngol 33: 57-59. 
7. Meriggioli MN, Donald BS (2004) Myasthenia gravis: Diagnosis. Seminars in Neurology. 24: 31-39.

8. Lertchavanakul A, Gamnerisiri P, Hirunwiwatkul P (2001) Ice test for ocular myasthenia gravis. J Med Assoc Thai 84(Suppl 1): S131-S136.

9. Golnik KC, Pena R, Lee AG, Eggenberger ER (1999) An ice test for the diagnosis of myasthenia gravis. Ophthalmology 106: 1282-1286.

10. Czaplinski A, Steck AJ, Fuhr P (2003) Ice pack test for myasthenia gravis: A simple, non-invasive and safe diagnostic method. J Neurol 250: 883-884.

11. Chatzistefanou KI, Kouris T, lliakis E, Piaditis G, Tagaris G, et al. (2009) The ice pack test in the differential diagnosis of myasthenic diplopia. Ophthalmology 116: $2236-2243$

12. Kubota T, Mishima H, Hanada M (1982) Paralytic dysphagia in cerebrovascular disorder-screening tests and their clinical application. General Rehabilitation 10: $271-276$.

13. Borenstein S, Desmedt J (1974) Temperature and weather correlates of myasthenic fatigue. Lancet 2: 63-66.

14. Borenstein S, Desmedt J (1975) Local cooling in myasthenia: Improvement of neuromuscular failure. Arch Neurol 32: 152-157.
15. Ricker K, Hertel G, Stodieck S (1977) Influence of temperature on neuromuscular transmission in myasthenia gravis. J Neurol 216: 273-282.

16. Saavedra JS, Femminini R, Kochen S, Ortiz de Zarate JC (1979) A cold test for myasthenia gravis. Neurology 29: 1075.

17. Seybold M (1986) The office tensilon test for ocular myasthenia gravis. Arch Neurol 43: 84-843.

18. Pascuzzi RM (2003) The edrophonium test. Semin Neurol 23: 83-88.

19. Sanders DB (2002) Clinical impact of single-fiber electromyography. Muscle Nerve Suppl 11: S15-S20.

20. Almeida DF, de Radeli RF, Melo Jr AC (2008) Ice pack test in the diagnosis of myasthenia gravis. Arq Neuropsiquiatr 66: 96-98.

21. Howard JF, Sanders DB, Massey JM (1994) The electrodiagnosis of myasthenia gravis and the Lambert-Eaton myasthenic syndrome. Neuro Clin 12: 305-329.

22. Programme for Neurological Diseases and Neuroscience (2004) Atlas Country resources for neurological disorders, World Health Organization, Geneva and World Federation of Neurology, London. Accessed on 20 March, 2017. 